\title{
Correlation Between Duration From Injury and Bone Mineral Density in Individuals With Spinal Cord Injury
}

\author{
Hyehoon Choi, $\mathrm{MD}^{1}$, So-youn Chang, $\mathrm{MD}^{2}$, Jaewan Yoo, $\mathrm{MD}^{1}$, Seong Hoon Lim, MD, $\mathrm{PhD}^{1}$, \\ Bo Young Hong, $\mathrm{MD}, \mathrm{PhD}^{1}$, Joon Sung Kim, $\mathrm{MD}, \mathrm{PhD}^{1}$
}

\begin{abstract}
${ }^{1}$ Department of Rehabilitation Medicine, St. Vincent's Hospital, College of Medicine, The Catholic University of Korea, Suwon; ${ }^{2}$ Department of Rehabilitation Medicine, Daejeon St. Mary's Hospital, College of Medicine, The Catholic University of Korea, Daejeon, Korea
\end{abstract}

Objective To investigate the correlation between bone mineral density (BMD) and duration of injury in individuals with spinal cord injury (SCI).

Methods Patients with SCI who visited the outpatient department between January 2009 and January 2019 were enrolled. Patients' most recent dual energy X-ray absorptiometry images were reviewed. According to the 2007 International Society for Clinical Densitometry guidelines, vertebrae with a local structural change were excluded when deriving spine BMD. If one or no vertebra is suitable for evaluation, spine BMD was judged as "improper for assessment". Correlation analysis was performed between duration from injury and BMD Z-scores of the hip and spine.

Results Among 83 individuals with SCI, the spines of 44 were judged as improper for assessment. The correlation analysis showed a significant negative relationship between the duration from injury and femur neck BMD ( $\mathrm{r}=-0.40$, $\mathrm{p}<0.01)$ and total proximal femur BMD $(\mathrm{r}=-0.39, \mathrm{p}<0.01)$. However, no significant correlation was found between the duration from injury and spine BMD Z-score.

Conclusion The duration of SCI correlated with hip BMD, but not with spine BMD. Further, more than half of the individuals with SCI could not undergo spinal assessment due to local structural changes. Therefore, spine BMD measurement is not an appropriate method for predicting future fracture risk in those with SCI.

Keywords Spinal cord injuries, Bone density, Osteoporosis

Received July 30, 2020; Revised August 17, 2020; Accepted September 3, 2020; Published online February 9, 2021

Corresponding author: Joon Sung Kim

Department of Rehabilitation Medicine, St. Vincent's Hospital, College of Medicine, The Catholic University of Korea, 93 Jungbu-daero, Paldal-gu, Suwon 16247, Korea. Tel: +82-31-249-7650, Fax: +82-31-251-4481, E-mail: svpmr@catholic.ac.kr

ORCID: Hyehoon Choi (https://orcid.org/0000-0002-1470-2914); So-youn Chang (https://orcid.org/0000-0003-0040-8084); Jaewan Yoo (https://orcid. org/0000-0002-4682-5716); Seong Hoon Lim (https://orcid.org/0000-0002-5475-4153); Bo Young Hong (https://orcid.org/0000-0001-9290-6173); Joon Sung Kim (https://orcid.org/0000-0001-7457-593X).

(c) This is an open-access article distributed under the terms of the Creative Commons Attribution Non-Commercial License (http://creativecommons.org/ licenses/by-nc/4.0) which permits unrestricted noncommercial use, distribution, and reproduction in any medium, provided the original work is properly cited. Copyright $\odot 2021$ by Korean Academy of Rehabilitation Medicine 


\section{INTRODUCTION}

It is established that bone loss occurs in individuals with spinal cord injury (SCI) [1-3]. Bone loss occurs immediately after injury and persists in the chronic phase [1]. However, whether bone loss occurs at the spine remains controversial [4,5]. In 1988, Biering-Sorensen et al. [4] studied 26 SCI individuals at 2-25 years after injury and found that the bone mineral contents of the femoral neck, shaft, and proximal tibia were low. However, their spine bone mineral density (BMD) was in the normal range. They inferred that the reason for this was that weight loading was applied to the spine while sitting [4]. Since then, Jaovisidha et al. [5] have made a counterargument that the risk of fracture of the spine in SCI individuals may be misinterpreted or underestimated because spine BMD measurements could appear higher due to various secondary progressive skeletal abnormalities, particularly neuropathic spondylopathy $[5,6]$. The bone in individuals with SCI undergoes various changes, including osteopenia, osseous, and articular soft tissue alternation. These changes may lead to spinal column abnormalities, such as disk space loss, bone sclerosis, fragmentation, osteophytosis, and subluxation. In their study [5], $49 \%$ of the vertebrae of individuals with SCI were abnormal with neuropathic spondylopathy, degenerative disease, and other diseases. According to Resnick and Niwayama [6], a small osteophyte $(0.5 \mathrm{~cm})$ can change the BMD by $1.54 \%-12.8 \%$. In a study comparing SCI individuals with an able-bodied population, 55\% of SCI individuals had mild to moderate degenerative joint disease and $25 \%$ of SCI individuals had severe degenerative joint disease in the lumbar spine, where as $40 \%$ of the control group had mild degenerative joint disease and none of them had moderate or severe degenerative joint disease [7].

In 2007, the International Society for Clinical Densitometry (ISCD) held position development conferences and established standards and guidelines for measuring skeletal health [7]. The ISCD is a multidisciplinary, nonprofit organization providing a central resource for a number of scientific disciplines with an interest in the assessment of skeletal health. According to the guidelines made as a result of those conferences in 2007, when checking BMD of the spine, physicians should use the posterior-anterior L1-L4 images of dual-energy X-ray absorptiometry (DXA) and exclude any vertebrae that are affected by local struc- tural changes or artifacts. "Artifact" may include surgical clips, navel rings, barium sulphate, metal from zippers, coins, clips, or other metallic objects. Local structural changes include osteophytes, syndesmophytes, compression fractures, and aortic calcification. If one or no vertebra level remains evaluable after excluding other vertebrae, the diagnosis should be based on a different valid skeletal site. For the hip region, the use of the femoral neck or total proximal femur is recommended [7]. The abovementioned contents were maintained in the latest 2019 ISCD Official Positions.

Cirnigliaro et al. [1] reported a marked bone loss in the distal femur and proximal tibia, as well as the femoral neck and total hip in SCI patients. Although the DXA score can predict the general fracture risk, the DXA score at a particular site can predict the fracture risk of that particular site more precisely [8]. Abderhalden et al. [9] showed that the DXA hip score predicted the fracture risk in SCI patients, but not the spine DXA score. The 2019 ISCD Official Position suggested that SCI adults with permanent motor and sensory disturbances should undergo a DXA scan of the total hip, proximal tibia, and distal femur [10]. There are many studies about BMD assessment in SCI patients, but no studies have analyzed this using the 2007 ISCD guidelines.

The aim of the present study was to describe whether there was a significant correlation between BMD of the hip or spine and the duration from injury in SCI individuals when the spine BMD was adjusted according to the ISCD guidelines. In addition, we aimed to assess whether spine BMD is appropriate as a predictor of fracture risk in SCI individuals.

\section{MATERIALS AND METHODS}

SCI individuals who visited the outpatient department between January 2009 and January 2019 were enrolled. Among them, individuals who had undergone DXA more than once were included. Those who were diagnosed with osteopenia or osteoporosis prior to the onset of disability and those who had bone disease were excluded. The present study was reviewed and approved by the Institutional Review Board of St. Vincent's Hospital (No. VC19RESI0005). The requirement for obtaining patients' informed consent was waived by the board.

Basic information, such as age, duration from injury (at 
the time of taking a DXA), injury level, and the American Spinal Injury Association (ASIA) impairment scale, was obtained through a medical record review. The most recent DXA images were reviewed by two readers. The readers had undergone a clinical densitometry course organized by the Korean Society for Bone and Mineral Research. Two readers independently read the same images. If there was a difference in the reading between the two readers, a decision was made by consensus. Interrater reliability was assessed using an intraclass correlation coefficient. To exclude the effects of age, sex, race, height, and weight, which are commonly known to affect BMD, we used a Z-score, to make comparisons with the general population with the same age, sex, race, height, and weight. According to the 2007 ISCD guidelines [7], the vertebral levels with local structural changes were excluded when calculating the "corrected L-spine BMD Zscore" (Fig. 1).

We named the L-spine Z-score before modification as "initial BMD Z-score" according to the guideline. When we checked the initial spine $\mathrm{Z}$-score, we excluded the vertebrae with an artifact such as the plate used for surgery. The reason for this was that BMD of a vertebral level with an artifact was not recorded in the DXA image that we reviewed. If one or no vertebra was suitable for evaluation due to local structural changes, the BMD of the whole vertebrae was deemed "improper for assessment". Consequently, according to the 2007 ISCD guidelines [7], we used the femur neck and total proximal femur BMD for assessing hip BMD. We analyzed the correlation again after categorizing individuals into non-ambulatory and ambulatory groups. Individuals who could walk with a device or assistance were allocated to the ambulatory group.

SPSS software version 12.0 (SPSS Inc., Chicago, IL, USA) was used for all data analyses. A correlation analysis was performed between the duration from injury and the BMD Z-score of the femur neck, total proximal femur, initial spine, and corrected spine. When the number was $<30$, a normality analysis was performed. When the assumption of a normal distribution was possible, a Pearson correlation coefficient was used. Corresponding missing values were removed. A $p$-value of $<0.05$ was considered statistically significant.

\section{RESULTS}

Eighty-four individuals with SCI had DXA. According to the exclusion criteria, one person who had heterotopic ossification was excluded. Thus, a total of 83 individuals were enrolled for this study. The mean duration from injury was $6.29 \pm 7.31$ years. The mean age was $51.6 \pm 15.3$ years. The other demographic characteristics of the individuals are shown in Table 1.

The mean initial spine BMD Z-score was $0.72 \pm 1.63$ with a corrected spine BMD Z-score of $0.30 \pm 1.4$, femur neck BMD Z-score of $-0.66 \pm 1.63$, and total proximal femur BMD Z-score of $-0.90 \pm 1.80$. The intraclass correlation coefficient for corrected spine BMD Z-score among readers was 0.98 (95\% confidence interval, 0.95-0.99), indicating an "excellent" level of agreement between readers.

The results of the correlation analysis revealed no significant correlation between the initial spine Z-score and duration from injury. We found significant negative correlations between duration from injury and femur neck BMD Z-score $(\mathrm{r}=-0.40, \mathrm{p}<0.01)$ and between duration
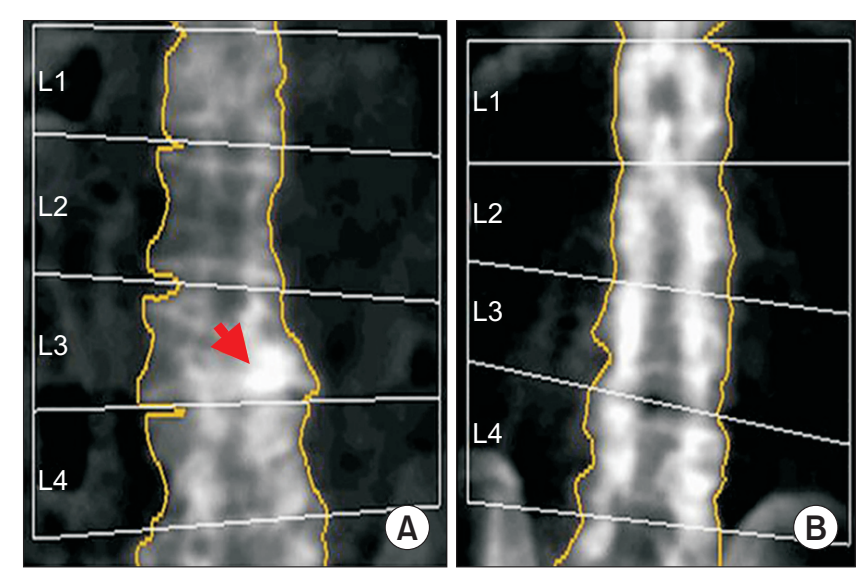

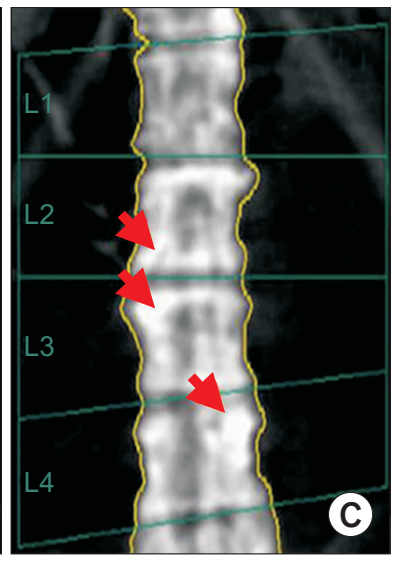

Fig. 1. Spine bone mineral density (BMD) images of spinal cord injury (SCI) individuals. (A) The L3 vertebra of the case 1 shows local structural changes (arrow). In this case, spine BMD is calculated using the $\mathrm{L} 1, \mathrm{~L} 2$, and $\mathrm{L} 4$ vertebrae. (B) Case 2 has L2-L5 laminectomy. (C) The L2, L3, and L4 vertebrae of case 3 show local structural changes (arrows). 
Table 1. Demographic characteristics of the participants

\begin{tabular}{|c|c|}
\hline Characteristic & Value \\
\hline \multicolumn{2}{|l|}{ Sex } \\
\hline Female & 23 \\
\hline Male & 60 \\
\hline Age (yr) & $51.60 \pm 15.30$ \\
\hline$<50$ & 38 \\
\hline$>50$ & 45 \\
\hline \multicolumn{2}{|l|}{ Neurologic level of injury } \\
\hline Cervical & 46 \\
\hline Thoracic & 31 \\
\hline Lumbar & 6 \\
\hline \multicolumn{2}{|l|}{ Mobility } \\
\hline Non-ambulatory & 49 \\
\hline Ambulatory & 34 \\
\hline \multicolumn{2}{|l|}{ ASIA impairment scale } \\
\hline A & 25 \\
\hline B & 11 \\
\hline $\mathrm{C}$ & 18 \\
\hline $\mathrm{D}$ & 29 \\
\hline Duration from injury (yr) & $6.29 \pm 7.31$ \\
\hline$<2$ & 33 \\
\hline $2-10$ & 28 \\
\hline$>10$ & 22 \\
\hline
\end{tabular}

Values are presented as average \pm standard deviation. ASIA, American Spinal Injury Association.

Table 2. Correlation between duration from injury and BMD Z-score

\begin{tabular}{lcc}
\hline \multicolumn{1}{|c}{ BMD Z-score } & $\begin{array}{c}\text { Correlation with } \\
\text { duration from } \\
\text { injury (r) }\end{array}$ & p-value \\
\hline Initial spine $(\mathrm{n}=83)$ & 0.17 & 0.13 \\
\hline Corrected spine $(\mathrm{n}=39)$ & -0.16 & 0.40 \\
\hline Femur neck $(\mathrm{n}=83)$ & $-0.40^{*}$ & $<0.01$ \\
\hline Total proximal femur $(\mathrm{n}=83)$ & $-0.39^{*}$ & $<0.01$ \\
\hline
\end{tabular}

BMD, bone mineral density.

*Pearson correlation coefficient is significant at 0.05 level.

from injury and total proximal femur BMD Z-score ( $\mathrm{r}=$ $-0.39, \mathrm{p}<0.01$ ). According to the 2007 ISCD guidelines [7], the spines of 44 individuals were evaluated as "improper for assessment". The correlation analysis of the remaining 39 individuals who had proper vertebrae for assessment showed no significant relationship between the corrected spine Z-score and duration from injury (Table 2).
Table 3. Correlation between duration from injury and BMD Z-score in non-ambulatory patients

\begin{tabular}{lcc}
\hline \multicolumn{1}{c}{ BMD Z-score } & $\begin{array}{c}\text { Correlation with } \\
\text { duration from } \\
\text { injury (r) }\end{array}$ & p-value \\
\hline $\begin{array}{l}\text { Initial spine BMD } \\
\text { Z-score (n=49) }\end{array}$ & 0.16 & 0.29 \\
$\begin{array}{c}\text { Corrected spine BMD } \\
\text { Z-score (n=21) }\end{array}$ & 0.34 & 0.13 \\
$\begin{array}{c}\text { Femur neck BMD } \\
\text { Z-score (n=49) }\end{array}$ & $-0.40^{*}$ & $<0.01$ \\
$\begin{array}{c}\text { Total proximal femur BMD } \\
\text { Z-score (n=49) }\end{array}$ & $-0.44^{*}$ & $<0.01$ \\
\hline
\end{tabular}

BMD, bone mineral density.

*Pearson correlation coefficient is significant at 0.05 level.

Table 4. Correlation between duration from injury and BMD Z-score in ambulatory patients

\begin{tabular}{lcc}
\hline \multicolumn{1}{c}{ BMD Z-score } & $\begin{array}{c}\text { Correlation with } \\
\text { duration from } \\
\text { injury (r) }\end{array}$ & p-value \\
\hline $\begin{array}{c}\text { Initial spine BMD } \\
\text { Z-score (n=34) }\end{array}$ & 0.16 & 0.39 \\
$\begin{array}{c}\text { Corrected spine BMD } \\
\text { Z-score (n=16) }\end{array}$ & 0.22 & 0.40 \\
$\begin{array}{c}\text { Femur neck BMD } \\
\text { Z-score (n=34) }\end{array}$ & $-0.41^{*}$ & 0.02 \\
$\begin{array}{c}\text { Total proximal femur BMD } \\
\text { Z-score (n=34) }\end{array}$ & $-0.36^{*}$ & 0.03 \\
\hline
\end{tabular}

BMD, bone mineral density.

*Pearson correlation coefficient is significant at 0.05 level.

For the non-ambulatory group, the duration from injury did not show any significant correlation with the initial spine Z-score. However, the duration from injury was significantly correlated with femur neck BMD Z-score ( $\mathrm{r}=$ $-0.40, \mathrm{p}<0.01)$ and total proximal femur BMD Z-score $(\mathrm{r}=$ $-0.44, \mathrm{p}<0.01)$. Among the 49 non-ambulatory individuals, one had no spine DXA image. The spines of 27 individuals were evaluated as "improper for assessment". On the normality test, the remaining 21 individuals showed a normal distribution. The duration from injury was not significantly correlated with the corrected spine Z-score (Table 3).

In 34 ambulatory individuals, the duration from injury was significantly correlated with the femur neck BMD Zscore $(r=-0.41, p=0.02)$ and total proximal femur BMD $\mathrm{Z}$-score ( $\mathrm{r}=-0.36, \mathrm{p}=0.03)$, but not with the initial spine 
BMD Z-score. The spines of 16 individuals were suitable for assessment and showed a normal distribution. The Pearson correlation analysis showed that the corrected spine Z-score was not significantly correlated with the duration from injury (Table 4).

\section{DISCUSSION}

In this study, the duration from injury was significantly and negatively correlated with the hip BMD Z-score but not with the initial spine BMD Z-score. After modifications based on the 2007 ISCD guidelines [7], the corrected spine BMD also did not correlate with the duration from injury. One reason for this might be the weight loading at the spine while sitting, as described by Biering-Sorensen et al. [4]. If that is the case, hip and spine BMD should have the same tendency in ambulatory individuals because they also had weight loading. However, this was not the case in the present study. The reason for this might be that the ambulatory SCI individuals could not walk as well as the general population does. In this study, we classified the individuals who used walkers or canes for ambulation into the ambulatory group. Second, we could not exclude all neuropathic spondylopathies. According to the 2007 ISCD guidelines, those with clearly abnormal vertebrae were excluded from this study. However, those with mild neuropathic or degenerative changes were not excluded. Spine BMD might appear higher owing to the influence of mild to moderate spondylopathy. Jiang et al. [2] reported that degenerative spine joints in SCI individuals can lead to stability of the spine and prevent vertebral fracture. Unlike primary osteoporosis where bone loss mainly occurs in the spine and hip, 50\% of fractures occur in the distal femur or proximal tibia and $10 \%-20 \%$ of fractures occur in the proximal femur in SCI individuals [11].

Spine BMD was not suitable for assessment in 43 of 83 SCI individuals, which is more than half of the analyzed individuals. This means that it is difficult to evaluate the spine BMD accurately owing to the neuropathic spondylopathy and degenerative changes, even if bone loss exists in the spine. In SCI individuals, spine BMD not only is preserved pathophysiologically, but also is difficult to assess accurately. Therefore, spine BMD is not appropriate as a predictor of fracture risk in individuals with SCI. $\mathrm{BMD}$ of the distal femur or the proximal tibia at the site of the fracture may be the most appropriate measurement, not spine BMD.

Several researchers have attempted to establish a protocol to measure the BMD of the distal femur and proximal tibia. Cervinka et al. [12] investigated a fragility fracture risk assessment algorithm in chronic SCI and suggested that we should use a T-score or Z-score of the hip or knee BMD in accordance with the recommendations of the ISCD and record the number of SCI-specific risk factors for fracture. However, it is difficult to derive the T-scores or Zscores of the distal or proximal femur because of the lack of large data on BMD in the general population. "Fracture threshold" is not a broadly used notion in primary osteoporosis, although it is useful for assessing fracture risk in regions where significant BMD data of the general population is not available. Eser et al. [13] reported that the fractures occur when femur BMD is $<114 \mathrm{mg} / \mathrm{cm}^{3}$ and tibial distal epiphysis is $<72 \mathrm{mg} / \mathrm{cm}^{3}$ as measured by quantitative computed tomography (qCT) [13]. Studies have investigated whether the distal femur or proximal tibia BMD is more appropriate when measured using DXA. Morse et al. [14] compared distal femur and proximal tibia BMD and found that the distal femur has better accuracy than the proximal tibia. Despite these studies, the spine and proximal hip are still being used to measure BMD. Furthermore, the bone around the knee has not yet been assessed for the prediction of fracture risk in SCI individuals.

This study has some limitations. First, the study design was not prospective; thus, we could not conclude that BMD of the femur decreased with time and spine BMD did not. We excluded only the vertebral levels with severe structural changes. Thus, we could not exclude the effects of the mild to moderate structural changes.

Many studies on SCI have used a nonparametric method because of the small number of subjects analyzed. However, we used the parametric method because we had a sufficient number to do so, and we believe that this is the strength of this study. Unlike other studies that have used qCT or DXA images in the lateral view, we used DXA images in the anteroposterior view, which are broadly used. We believe that our findings will support future studies. We also highlighted the need for appropriate methods for the assessment of BMD of the knee or distal femur, which are the most common fracture sites in SCI individuals, to establish fracture risks.

In conclusion, this study has highlighted the difficulty 
of assessing spine BMD accurately in SCI individuals. We also showed that the duration from injury correlates significantly with hip BMD, but not with spine BMD, after adjusting for the effect of spondylopathy. Therefore, spine BMD measurement in SCI individuals is not an appropriate method for predicting future fracture risk. Further research is needed to elucidate the most relevant site for BMD measurements in SCI patients.

\section{CONFLICT OF INTEREST}

No potential conflict of interest relevant to this article was reported.

\section{AUTHOR CONTRIBUTION}

Conceptualization: Kim JS. Methodology: Choi H, Kim JS. Formal analysis: Choi H, Yoo J, Chang S. Project administration: Kim JS. Writing - original draft: Choi H. Writing - review and editing: Hong BY, Lim SH. Approval of final manuscript: all authors.

\section{REFERENCES}

1. Cirnigliaro CM, Myslinski MJ, Asselin P, Hobson JC, Specht A, La Fountaine MF, et al. Progressive sublesional bone loss extends into the second decade after spinal cord injury. J Clin Densitom 2019;22:185-94.

2. Jiang SD, Dai LY, Jiang LS. Osteoporosis after spinal cord injury. Osteoporos Int 2006;17:180-92.

3. Jiang SD, Jiang LS, Dai LY. Mechanisms of osteoporosis in spinal cord injury. Clin Endocrinol (Oxf) 2006;65:555-65.

4. Biering-Sorensen F, Bohr H, Schaadt O. Bone mineral content of the lumbar spine and lower extremities years after spinal cord lesion. Paraplegia 1988;26:293301.

5. Jaovisidha S, Sartoris DJ, Martin EM, De Maeseneer M, Szollar SM, Deftos LJ. Influence of spondylopathy on bone densitometry using dual energy X-ray absorptiometry. Calcif Tissue Int 1997;60:424-9.
6. Resnick D, Niwayama G. Degenerative disease of the spine. In: Resnick D, editor. Diagnosis of bone and joint disorders. 3rd ed. Philadelphia, PA: Saunders; 1995. p. 1372-462.

7. Lewiecki EM, Gordon CM, Baim S, Leonard MB, Bishop NJ, Bianchi ML, et al. International Society for Clinical Densitometry 2007 Adult and Pediatric Official Positions. Bone 2008;43:1115-21.

8. Zleik N, Weaver F, Harmon RL, Le B, Radhakrishnan R, Jirau-Rosaly WD, et al. Prevention and management of osteoporosis and osteoporotic fractures in persons with a spinal cord injury or disorder: a systematic scoping review. J Spinal Cord Med 2019;42:735-59.

9. Abderhalden L, Weaver FM, Bethel M, Demirtas H, Burns S, Svircev J, et al. Dual-energy X-ray absorptiometry and fracture prediction in patients with spinal cord injuries and disorders. Osteoporos Int 2017;28:925-34.

10. Morse LR, Biering-Soerensen F, Carbone LD, Cervinka T, Cirnigliaro CM, Johnston TE, et al. Bone mineral density testing in spinal cord injury: 2019 ISCD Official Position. J Clin Densitom 2019;22:554-66.

11. Edwards WB, Schnitzer TJ. Bone imaging and fracture risk after spinal cord injury. Curr Osteoporos Rep 2015;13:310-7.

12. Cervinka T, Lynch CL, Giangregorio L, Adachi JD, Papaioannou A, Thabane L, et al. Agreement between fragility fracture risk assessment algorithms as applied to adults with chronic spinal cord injury. Spinal Cord 2017;55:985-93.

13. Eser P, Frotzler A, Zehnder Y, Denoth J. Fracture threshold in the femur and tibia of people with spinal cord injury as determined by peripheral quantitative computed tomography. Arch Phys Med Rehabil 2005;86:498-504.

14. Morse LR, Lazzari AA, Battaglino R, Stolzmann KL, Matthess KR, Gagnon DR, et al. Dual energy x-ray absorptiometry of the distal femur may be more reliable than the proximal tibia in spinal cord injury. Arch Phys Med Rehabil 2009;90:827-31. 